\title{
EINLEITUNG DES HERAUSGEBERS
}

\section{Zur allgemeinen Würdigung der "Dialektik“ Schleiermachers}

Dem Rausch des Höhenfluges ins Absolute steht Schleiermacher mit sokratischer Nüchternheit und Ironie gegenüber. Unberührt von dem „Kontagium“ idealistischer Systemkonstruktionen, zählt er sich zu den Stillen, ,die anders philosophieren, aber damit nicht öffentlich auftreten, weil sie auf keinen Erfolg rechnen können, sondern das ihrige als eigenes Gut und eigene Art festhalten" (S. 8I dieser Ausgabe). In dieser Stille formt sich ihm nach den Gesetzen und Kräften eines "Gesamtbildes der menschlichen Kultur" in jahrelanger hingebungsvoller Arbeit die Wundergestalt eines Organons und Kriterions des Wissens: die „Dialektik“. - Gewiß, es fehlt diesem Werk alles, was es zu dem ideengeschichtlichen Kontinuum der nachkantischen Epoche in ein erträgliches Verhältnis setzen könnte: das Prometheische des absoluten Anfangs, die grandiose Unbedenklichkeit in der Preisgabe des Zeitlichkonkreten zugunsten abstrakter Ewigkeitsaspekte, der spekulative Hochmut eines in intellektueller Anschauung kontemplierenden „Aristokratismus der Intelligenz", kurzum die himmelstürmende Selbstsicherheit des System-Pathos. Aber diese Beschränkung erklärt sich nicht aus spekulativem Unvermögen, sondern aus einer unbedingten Ehrfurcht vor der Fülle des Lebendigen. Schleiermachers anthropologisch verdichtetes Denken wurzelt breit und tief im Diesseitsgrunde seines geschichtlichen Menschseins. „Die Menschheit in sich zu betrachten, und, wenn man einmal sie gefunden, nie den Blick von ihr zu verwenden, ist das einzige sichere Mittel, von ihrem heiligen Boden nie sich zu verirren" (Monologen. Ausg. SchieleMulert. S. 27). Das war die wundersame, nichtverstandene Neujahrsgabe an das Jahrhundert. Den Reichtum an indi- 
viduellen Gestaltungen, wie er aus diesem Boden emporquillt, mit dem Leibniz-Instinkt für das chargé du passé et gros de l'avenir enthusiastisch anzuschauen und in seinen tausendfältigen Verästelungen aufzudecken, erscheint ihm als vornehmste Aufgabe des Philosophen. So weiß er um die zeit- und volkbedingte Natur von Religionen und metaphysischen Systemen. Er weiß, daß alles organische Wissen nur dann ein Ganzes ist, wenn es in der Eigentümlichkeit seines Wachstums erhalten bleibt; daß die Weltvorstellung „für jeden Punkt, von dem sie ausgeht, eine andere“, individuell und national gebundene, sein muß; und er erblickt in dem tollkühnen Überstieg ins Zeitlos-Allgemeine die große Verkehrtheit idealistischer Systemgestaltungen (vgl. Sittenlehre. Ausg. Otto Braun. Leipzig 1927. S. 167).

Zum Seinsverständnis des unerhörten Reichtums von Menschheit bedarf es gänzlich neuer Zurichtungen des Denkens. Und wenn die Dialektik die Logik zur Bodenstiftung von anthropologischer Metaphysik bemüht, so ist es nicht der erstorbene Logos des reinen Gedankens, dem eine erborgte Dynamik zum Scheinleben verhelfen muß: es ist der Dialogos der lebendigen Wechselrede, der bis in seine letzten Stammformen hinab die Eigentümlichkeit seines individuellen Lebens bewahrt. Am Anfang steht die Rede, nicht der Begriff. Die Rede ist Ausdruck und Urtatsachemenschlicher Vernunft als ewigen Wissenwollens./Und das Dynamische an ihr, das also, was sie zum Prototyp der Lebensoszillationen menschlichen Geistes macht, schreibt sich weder aus dem reinen Denken noch aus der stumpfen Sinnlichkeit her, sondern hat seinen Grund in dem geschichtlich gewachsenen, beide Pole zu organischer Spannungseinheit verbindenden Körper der Sprache. Dank dieser Wurzelung im Sprachgrunde ist sich menschliche Vernunft zugleich des einwohnenden Seins Gottes bewußt. Für Schleiermacher wie für Meister Eckhart ist Gott ,,rede der redenden. Dar umbe ist er aller natûren natüre" (Meister Eckhart. Ausg. F. Pfeiffer. S. 540). Das Reich der Wahrheit, ,wie sie ohne Hülle an und für sich selbst ist" (Hegel), ist auch für Schleiermachers Dialektik das immerdar aufgegebene und gesuchte Ziel; ein Ziel jedoch, vor dem jede menschliche Rede verstummen muß. Gewiesen ist uns allein der Weg dorthin, der 
uns niemals aus dem Felde der relativen Identität hinauszuführen vermag. In diesem Feld der Endlichkeit des Erkennens das Methodenbewußtsein zu stärken, zu lehren, wie wir in peinlicher Rechenschaftsablegung bei jedem Fortgang des Denkens der Gefahr eines bodenlosen Relativismus begegnen, und den Einbruchsstellen der Sünde des Irrtums nachzuspüren, ist das eigentliche kritische Geschäft der Dialektik.

Nun war der Ruf nach einer solchen Disziplin, die das Verhältnis von Spekulation und Empirie regelt, von einem der idealistischen Systembildner selbst ausgegangen. In den „Vorlesungen über die Methode des akademischen Studiums" erklärt Schelling, daß es ohne dialektische Kunst keine wissenschaftliche Philosophie gebe, weil anders nicht "die Ungeheuer einer rohen dogmatischen Philosophie" erschlagen werden können (Schelling WW. V. S. 267). In dunkler Ahnung schreibt er der Logik die Fähigkeit zu, unter dem Namen "Dialektik“ als reine Kunstlehre der Philosophie auftreten zu können (ebda. S. 269). Doch existiere sie noch nicht, weil die traditionelle Logik in der "Sphäre der Endlichkeit" befangen sei und alle wahre Wissenschaft des Menschen ,in der Idee des Menschen, also überhaupt nicht in dem wirklichen und empirischen Menschen" gesucht werde. Wir sehen hieraus, wie es mit der „empirischen Konstruktion“ bei Schelling bestellt ist. Während bei ihm das Empirische sogleich wieder ins Ideale sublimiert wird und die Philosophie erst mit dem aufgehobenen Gegensatz zwischen Spekulation und empirischem Wissen beginnt, hat sie nach Schleiermacher im Akt des werdenden, aber nie yollendeten Aufhebens des Gegensatzes ihr Leben und bleibt immer mit der bunten Fülle der Lebensoszillationen des wirklichen Menschseins verbunden. Wenn Schleiermacher 1804 in der Rezension von Schellings ;Vorlesungen“ darauf hinweist, ,wie genau die historische Konstruktion (der Sprache) mit der der idealen Welt selbst zusammenhängt, wie alles Historische in Künsten und Wissenschaften sich in der Sprache abspiegelt und nur in Verbindung mit ihr recht zu erkennen ist" (Leben i. Br. IV. S. 59I), so steht ihm das Programm seines eigenen methodischen Unternehmens bereits lebendig vor Augen. Die 
Dialektik wird zur Kunst der spekulativen menschlichen Rede, wobei sie sich aus den grammatikalischen Grundgemeinsamkeiten des Sprachleibes die metaphysischen Prinzipien der Konstruktion alles Wissens, aus den logischen Verknüpfungsgesetzen die kritischen Kanones für den Oszillationsproze $\beta$ des scheiternden, aber immer schon in der Wahrheit stehenden Dialogos herausarbeitet.

Ist nun die „Dialektik“ der Anlage nach eine Summe von Regeln und Lehrstücken, nach denen gedacht werden soll? Steht sie also als Leitfaden außerhalb des Bereiches der Kunst, von der sie selbst Lehre sein will? - Gewichtige Gründe scheinen für eine bejahende Antwort zu sprechen. Drückt sich doch die imperativische oder wenigstens konsultative Absicht schon in dem Titel einer „Kunstlehre des Denkens" aus; bekunden zudem die in knappe Paragraphen eingezwängten Leitsätze des Grundheftes von 1814, auf die Schleiermacher auch später noch zurückgreift, deutlich genug das auf lehrhafte Statik zielende "Geschäft" des Dialektikers; und zeigt endlich der formale Teil eine unverkennbare Neigung, sich zu scharf formulierten „Kanones“ mit imperativischer Satzform zu verdichten. Danach also handelte es sich in der Dialektik um eine zur Beendigung des Streits gegebene "Anweisung“, die dem Streit selbst souverän gegenübersteht.

Nun hat Schleiermacher andrerseits keinen Zweifel darüber gelassen, $\mathrm{da} B$ er eine solche Anweisung ,als eine ursprünglich hervorbringende oder didaktische" (S. 38 dieser Ausg.) für durchaus verfehlt hält und da $\beta$ jedes System von Sätzen, das eine „Wissenschaft des Wissens" aufzustellen vorgibt, in der Luft schwebt. Mit anderen Worten: Dialektik, sofern sie „als eine Gegebenes prüfende oder kritische Anweisung " (S. 38 dieser Ausg.) sein will, macht sich weder anheischig, ,das Wissen aus schlechthin neuen Anfängen zu entwickeln" (S. 37 dieser Ausg.), noch von einem bestimmten Punkte des Denkprozesses ab streitfreies Denken weiterzuentwickeln, noch endlich gar einzelne Sätze des Denkgeschehens als streitfreie herauszupräparieren, während andere noch im Streit stehen, um so allmählich aggregatartig Gewußtes anzuhäufen. Das Ende des Streites ist nicht an dem einen Punkt früher als an dem anderen. Es 
liegt in der ,zyklischen Natur" des Erkennens, daß es kein Erwerben im Gebiet des Wissens gibt, derart, „daß ein Wissen vom andern abgeschnitten wäre, sondern nur so, daß eine allmähliche Verklärung des Wissens entsteht, indem deutlicher, bestimmter, sicherer wird, was man auf einer niedrigen Stufe des Bewußtseins auch schon hatte" (Vorl. 1818. Dial. ed. Jon. S. I).

Schleiermacher hat die Gefahr eines Mißverstehens seiner Grundabsicht sehr wohl gesehen und sie mit verschiedenen Mitteln abzuwenden gesucht. Einige Male bedient er sich der Analogie mit den schönen Künsten (S. 39 u. 43 ff. d. A.). Auch für die ausübenden Künste gebe es „Kunstlehren“, die sich auf „negative Sätze“, d. h. auf Kautelen über das, „was vermieden werden soll", beschränken. In gleicher negativer Haltung stehe die Dialektik dem streitigen Denken gegenüber, indem sie aufzuzeigen habe, wo der Irrtum entstehe und wie er zu vermeiden sei. Schleiermacher aber hat zugleich keinen $Z$ weifel darüber gelassen, da $B$ ein solcher Vergleich nur ein propädeutischer Notbehelf sei, ja da $B$ er im Grunde dem tieferen Verständnis der Dialektik geradezu entgegenarbeite. Denn es ist das unterscheidende Merkmal der Dialektik gegenüber praktischen Kunstregeln, daß sie dem Gebiet, auf das die Regeln zielen, immanent sei. Die „Dialektik“" steht selbst im Zustand des Streites; sie höbe sich selbst auf, wenn sie sich als undialektische Aussage über Dialektik ausgeben wollte. Mit diesem dogmatischlehrhaften Zustand haben die "Lossagungen“ der Einleitung (S. 27 d. A.) ein für allemal abgerechnet.

$\mathrm{DaB}$ sich die Dialektik dennoch das Recht nehmen darf, "Anweisungen" zu geben, wird in der gleichen Einleitung letzter Hand, die für das Verständnis der Grundabsicht Schleiermachers von höchster Bedeutung ist und deshalb an die Spitze einer Neuausgabe gehört, eindringlich erörtert. Ausgehend von der ungeteilten Einheit unseres Menschseins entwickelt hier Schleiermacher eine Theorie von den drei Erscheinungsformen geistiger Tätigkeit, die sich als geschäftliches, künstlerisches und reines Denken äußern; doch so, daß sich niemals eine dieser Formen von der andern abtrennt, sondern da $B$ vielmehr alle drei dem Keime nach in jedem noch so primitiven Denkgeschehen vorhan- 
den sind (vgl. S. $20 \mathrm{ff}$. d. A.). Es steht also der „Reinheit“ des dialektischen Denkens, das „wesentlich immer gesprochen sein muß",(S. 24), nicht im Wege, wenn auch hier ein "Geschäft", nämlich das auf die „Selbigkeit des denkenden Seins" gerichtete Wissenwollen, getrieben wird und wenn „dialektische Regeln die Natur des geschäftlichen Denkens an sich tragen“ (S. 33). Doch fallen diese „Regeln" nicht wie reife Früchte als isolierte und statische Teilerkenntnisse aus dem Proze $B$ heraus; sie werden nur von der „Dialektik“, als dem Gespräch kat'exochen, mit den Prinzipien der Konstruktion des Wissens zugleich als Grundsubstanz jeder streitigen Rede zur Erscheinung gebracht und bleiben immer in das Ganze dieses Prozesses eingebettet. Die Relativität in der Wahl des Ansatzpunktes, der Einfluß der „besonderen Denkgeschichte des Wählenden“" (S. 37) und die Bindungen an den jeweiligen Sprachkreis sind die schicksalsmäßigen formativen Quellkräfte, die dem Wachstumsvorgang des dialektischen Geschehens seine individuelle Prägung verleihen.

Wir geben diesen Keimgedanken ihre programmatische Gestalt: Die Dialektik ist selbst eine Erscheinungsform des Sprachgeistes, dem sie das Leben ihrer Rede verdankt. Sie ist im wahrsten Sinne Organon, nicht, weil sie diesem Prozeß unbeteiligt gegenübersteht, sondern weil in ihr komplikativ alle Entfaltungsmöglichkeiten der spekulativen Rede angelegt und in einer höheren Potenz des Um-sich-selbstWissens dargestellt sind. Sie ist zugleich Kriterion und Kunstlehre des Sichverständigens, weil sie in jedem Dialogos das Gespräch des Gesprächs ist. Sie wächst auf dem Boden der individuellen Sprachgemeinschaft und beendet ihr Dasein in dem Augenblick, wo auch die Sprache ihren Geist aufgibt. Denn die Sprache hat ihrerseits auch das Leben vom Gespräch, dessen Vegetationsbasis wiederum der Streit und das Wissenwollen bilden. Sind einmal alle Zweifel gelöst und ist die Wahrheit erreicht, so ist die Sprache „vollkommen sich selbst gleich", ,fest" und ,absolut identisch geworden für alle, die sie reden " (Ausgabe Jonas. Beilage E [I83I] S. 480/8I). Aber niemand redet sie dann auch mehr, weil im absolut Selbstverständlichen das Sichverständigen überholt ist. Im „Identischwerden“ der 
Sprache liegt ihre Euthanasie und zugleich das Ende des Menschseins. Denn menschliche Existenz gründet im Gespräch. Nur auf dem Wege zur Wahrheit und im Prozeß des Wissenwollens blühen die kommunikativen Triebe, die dem Wesen menschlicher Gemeinschaft seinen Sinn geben. Nur im Miteinandersprechen über etwas ist die Möglichkeit des Zueinanderkommens offen. So hat Heidegger das Hölderlin-Wort „Seit ein Gespräch wir sind -“" gedeutet (M. Heidegger, Hölderlin und das Wesen der Dichtung. München 1937. S. 8). In dem gleichen Grunde wurzelt Schleiermachers Kunstlehre des Gesprächs, die, was immer auch im einzelnen beanstandet werden mag, von einer so erhabenen Größe des Entwurfs, von einer so staunenswerten Zielsicherheit in der Entfaltung ist, daß ich sie dem Größten gleichsetze, was auf dem Felde abendländischer Geistigkeit geschaffen wurde.

Es ist bekannt, welche Bedeutung Schleiermacher selbst seiner Arbeit an der Dialektik beigelegt hat und mit welcher Zähigkeit er ihr dreiundzwanzig Jahre hindurch treu geblieben ist. Nachdem der Entschluß zu einer Vorlesung ïber spekulative Philosophie lange in ihm ,gewurmt" "hatte, trägt er die Dialektik I8I I zum erstenmal an der Berliner Universität vor. Noch ist ihm keine gußfertige Form zur Hand, nur die "Hauptmassen" sind vorhanden und müssen sich unter den prometheischen Eingebungen des freien Vortrages zunächst notdürftig ordnen. Die ersten Aufzeichnungen können nicht befriedigen, Spannungen und Widersprüche zeigen sich allenthalben, und der organische Aufbau liegt noch völlig im argen. Es entstehen neue Entwürfe, in fünf weiteren Vorlesungsjahren geschaffen und an die früheren angeglichen. Und es verbindet sich damit der Wunsch, dieser Lieblingsdisziplin eine organische Gestalt zu geben, wie sie bereits die "Glaubenslehre“ gefunden hatte.

Wie wenig aber entsprach diesem unablässigen Bemühen die Wirkung nach außen! - Wäre je ein Lärm um das Werk entstanden und hätte sein Schöpfer die Rührigkeit der groBen Systembildner seiner Zeit besessen, so hätte man sich wohl ernsthafter mit seiner Philosophie auseinandergesetzt. So galt er - und gilt bei vielen auch heute noch - als Epi- 
gone Kants, als Mitläufer Fichtes oder Schellings; oder gar als Spinozist: Etikettierungen, von denen er mit bitterem Humor Kenntnis nahm.

Wenn man Schleiermacher immer wieder Mangel an Originalität vorgeworfen hat, so darf ich billigerweise an die Bemerkung erinnern, mit der er selbst die Methode Schellings in Schutz genommen hat: da $\beta$ ein solcher Vorwurf nur für den einer sei, „,der das rohe Aufnehmen fremder Gedanken nicht von einem solchen zu unterscheiden weiß, welches sich durch seine Gehörigkeit in ein regelmäßig aufgeführtes Ganze als ein wahres zweites Erfinden ankündigt, dem das Frühere eines anderen nur zufällig vorausgegangen ist" (Leben i. Br. IV. S. 586). Während aber bei Schelling die Anlehnungen vielfach mit Händen zu greifen sind, hat der Einschmelzungsproze $B$ bei Schleiermacher einen solchen Grad der Innigkeit angenommen, da $B$ er jedem rohen Zugriff des üblichen historischen Vergleichverfahrens spottet. Wir haben es hier, wie Wehrung (Die Dial. Schl.s. Tüb. 1920. S. 105) treffend bemerkt, mit der „Hinterlassenschaft eines der beweglichsten Geister der Weltgeschichte" zu tun, der, ,fremde Anregungen mit eigenen Forderungen und Einsichten aussöhnend, immer neue Beziehungen zwischen Getrenntem anzubahnen verstanden hat". - Doch, seltsam genug: der Eklektizismus Schellingscher Prägung hat dem philosophiegeschichtlichen Ruf seines Erfinders keinen Abbruch getan. Bei Schleiermacher dagegen kam man kaum je über die Abhängigkeitsschnüffelei hinaus und scheute mit traditioneller Hartnäckigkeit die „Anstrengung des Begriffs", um zu den Quellgründen seines originalen Denkens vorzudringen. Er ist nun einmal der Theologe von Beruf, der als Philosoph die Ressortschranken in dilettierender Weise überschreitet.

Wie ungerecht dieses Urteil ist, beweist einmal Schleiermachers philologisch-hermeneutischer Umgang mit den antiken Quellen der Spekulation, wodurch er sich ein von keinem der großen Systembildner je erreichtes problemgeschichtliches Wissen erwerben mußte, beweist vor allem seine lebenslange Auseinandersetzung mit Sokrates und Platon, zu der die umfangreiche Übersetzungstätigkeit an den platonischen Dialogen nur den äußeren Anstoß gegeben hat, 
während der eigentliche Grund in einer tiefinnerlichen Geistesverwandtschaft Schleiermachers mit den attischen Denkern zu suchen ist. Der „Phaidros“ liefert ihm das logische Rüstzeug für die lebendige Wechselrede, der "Sophistes“ mit seiner abgründigen Seinsdialektik wird ihm zur Kernzelle seines eigenen systematischen Entwurfes, der von hier aus das Wagnis unternimmt, Eleatismus und Heraklitismus zu einer polaren Spannungseinheit zusammenzuschließen. Der problemgeschichtlichen Forschung eröffnet sich hier ein fruchtbares Arbeitsgebiet; eine erste Orientierung habe ich in meinem Aufsatz: „Der Geist der Sokratik im Werke Schleiermachers" gegeben (Festschrift für Eduard Spranger. Leipzig 1942. S. I03 ff.).

Der Geist Platons läßt Spielraum für eine Unendlichkeit von Gestaltungen. Man verliert nicht seinen Eigenwuchs, wenn man sich von platonischer Gesinnung erfüllen läßt. Anders dagegen verhält es sich mit Kant. Man ist entweder Kantianer oder ist es nicht. Was dazwischen liegt, ist entweder tragisches Versagen oder peinliche Karikatur. In den Bereich des scheiternden Kantianismus hat man auch Schleiermacher zu stellen gesucht. Wenn ich dieser Logende entgegentrete, so muß ich mich hier darauf beschränken, die Achsenverschiedenheit beider Geistesräume kenntlich zu machen. Ich gehe nicht auf die zahlreichen Selbstbekenntnisse Schleiermachers ein, die von seiner grundsätzlichen Abneigung gegen die Kantische Philosophie zeugen. Es bleibt die Tatsache bestehen, da $\beta$ er sich mit dem ihm widerstrebenden Stoff der „Kritik“ gründlich auseinandergesetzt hat - bis zur scheinbaren Aneignung einiger terminologischer Bestandstücke, die ihn in der Ära des Neukantianismus zugleich interessant und verdächtig gemacht haben. Ist nicht mit der Kennzeichnung der organischen und intellektuellen Funktion der Dualismus der ZweiStämme-Theorie übernommen? Ist nicht die Anlehnung bis zur Assimilation der selbst bei Kant schon anrüchigen Lehre vom "Schematismus" gesteigert worden? Und kann es einen überzeugenderen Beweis geben für die Hybris, Kantianer zu spielen, als jenen berüchtigten \& I I9 der Dialektik von 1814, worin der Satz steht: „Ohne Einheit und Vielheit ist die Mannigfaltigkeit unbestimmt; ohne Mannigfaltigkeit ist 
die bestimmte Einheit und Vielheit leer"? Dazu gesellen sich noch andere angeblich pseudokritische „Kennzeichen“; etwa der Art: „Die Bestimmung, in der das Wissen ist, ist Spontaneität, die chaotische Masse des Eindrucks, in der das Wissen nicht ist, ist Rezeptivität" (\$ 274). Hier scheint es ja sonnenklar zu sein, daß Schleiermacher im Grunde nichts weiter wollte als Kant; daß vor allem sein ,unmittelbares Selbstbewußtsein " sich nicht wesentlich von der transzendentalen Apperzeption unterscheidet; und da $B$ alles andere, was nun allen Umdeutungsversuchen zum Trotz gar nicht mehr zum Kritizismus passen will, also der ganz und gar originale Schleiermacher, eben scheiternder Kantianismus ist.

Alle Auslegungen dieser Art übersehen, daß die Terminologie der kritischen Philosophie nicht erst von Kant geschaffen worden ist. Es ist daran zu erinnern, daß man lange vor aller transzendentallogischen Problematik von der Duplizität der Vermögen sprach; daß Tetens bereits die „Auflösung der Erkenntniskraft" in Rezeptivität und Aktivität vorgenommen und dem "Chaos der Empfindungen" den "Aktus des Bewußtseins" gegenübergestellt hatte, wodurch bewirkt werde, da $\beta$ wir diese Mannigfaltigkeit als ein ,,vereinigtes Ganze" empfinden (Philos. Versuche. S. 389. Vgl. S. 336). In allen grundsätzlichen Fragen steht Schleiermacher der Kantischen Lehre völlig ablehnend gegenüber. „Es gibt keine Trennung des Wesens der Dinge von ihrer Erscheinung“" (§ I73). Entschiedener kann die Absage an die Kritik der reinen Vernunft nicht ausgesprochen werden. Niemals später findet sich der obenerwähnte angeblich „kantische" Satz des § I I9 in dieser formelhaften Prägung. Und niemals hat er bei Schleiermacher den gegenstandskonstitutiven Sinn der Transzendentalkritik, sondern ausschließlich den Charakter eines Regulativs im Oszillationsproze $B$ des Wissens, dessen Wachstumsgrad als Durchdringungsintensität von Ordnung, Bestimmtheit der intellektuellen Funktion einerseits und von Fülle und Lebendigkeit der organischen Funktion andrerseits dadurch gekennzeichnet werden soll. Die Vorlesung von I818 drückt dies so aus: „Das Denken ist um so lebendiger, je mehr die organische Tätigkeit vorherrscht, es ist um so bestimmter, je mehr die 
Vernunfttätigkeit vorherrscht" (Nachschrift v. Bluhme. Vgl. S. 155 dieser Ausg.). - Vor allem aber ist auf die abgrundtiefe Kluft zu achten, die sich zwischen beiden Denkern bei der Frage nach dem Erkenntniskriterium auftut. Bei Kant handelt es sich um den Bereich des phänomenalen Seins, in dem Objektivität überhaupt erst begründet werden soll. Hier fließen die Charaktere der Allgemeingültigkeit und Notwendigkeit aus dem gemeinsamen Akt der Synthesis, und die Erkenntnisfrage ist nach der Entrechtung aller Transzendenz nach dem einen Brennpunkt der Einheit des. Bewußtseins gerichtet. Bei Schl. dagegen trägt der Wissensproze $\beta$ einen bipolaren Charakter: gleichmäßige Vollziehbarkeit in allen Denkenden und Übereinstimmung des Denkens mit dem Sein. Man glaube nicht etwa, da $B$ das zweiteKriterium jenen unverbindlichen formalen Sinn hat, in dem Kant die Wahrheit als Übereinstimmung einer Erkenntnis. mit ihrem Gegenstande definiert. In dem zwischen dem terminus a quo des Gottesgrundes und dem terminus ad quem der Welt ausgespannten Bogen bildet das „Reale“ einen ganz und gar selbständigen und unersetzbaren Pfeiler. Niemals hat Schleiermacher die Bipolarität des Kriteriums im kritischen Sinne korrigiert, am allerwenigsten im Vorlesungsjahr 1822, wovon die vorliegende Ausgabe auf Schritt. und Tritt Zeugnis ablegt. Hier gibt es keine Brücke zum Kritizismus, und es hat keinen Zweck zu fragen, wieweit Schleiermacher mit dem Kritizismus geht. Er geht nicht einen Schritt mit ihm, sondern steht ihm vom ersten Ansatz völlig selbständig gegenüber, in lebendiger Anknüpfung an die Tradition der rationalen Psychologie des 18. Jahrhunderts. Ich habe in meiner Darstellung der Ästhetik Schleiermachers darauf hingewiesen, in welchem Maße sich Schleiermacher dem Kantgegner Eberhard verbunden fühlt. Nicht minder deutlich treten die Beziehungen zu diesem Manne, der ihm die Platonliebe in die Seele gepflanzt hat, bei der Frage nach dem Zusammenhang zwischen Sinnlichkeit und Verstand hervor. Auch bei Eberhard liegen beide Vermögen nicht ,abgesondert und insuliert in der Seele", sondern gehen kontinuierlich ineinander über. Heißt es bei ihm: „Im Denken Einheit, im Empfinden Mannigfaltigkeit" (Allgemeine Theorie des Denkens und Empfin- 
dens. 1776. S. 68), so bei Schleiermacher: „In allem Denken ist die Vernunfttätigkeit der Quell der Einheit und Vielheit, die organische Tätigkeit der Quell der Mannigfaltigkeit" (Dial. 1814. Jon. \& 118). Geht nach Eberhard die Seele vom Anschauen der Sachen auf die Vorstellung der Zeichen über, bis sie sich „weiter nichts mehr als der Zeichen bewußt ist und mit einer blinden Operation in den tiefen Gängen der Wahrheit fortrückt" (ebda. S. II5), so finden wir in voller Ubereinstimmung hiermit bei Schleiermacher die Erklärung, daß, je mehr wir die realen Begriffe ihrer organischen Seite entkleiden und sie ,nur als Zeichen brauchen", wir um so weniger dabei denken (Dial. \& I Io). Diese Auffassung von dem gradweisen Ineinanderwirken beider Vermögen, die sich zu dem Eberhardschen Gesetz verdichtet: „Die Intensität der Einheit ist in ratione inversa der Mannigfaltigkeit und umgekehrt" (Allg. Th. S. 78), findet in Schleiermachers Theorie der Wissensbildung, in dem Beziehungsgefüge zwischen Schema und Formel ihre geniale Vertiefung und Fortbildung.

Mit solchen Hinweisen soll nun nicht etwa das Schelmenspiel getrieben werden, Schleiermacher aus einer Hörigkeit in die andere zu versetzen. Vor Kant wird man zum Epigonen, vor Eberhard nicht. Der eine ist eine Welt mit eigener Atmosphäre, der andere nur eine magere Scholle, die ihre Lebensluft aus dem Odem einer Epoche empfängt. Um die Größe des Entwurfes der „Dialektik“" abzuschätzen, bedarf es anderer Maßstäbe, als sie das 18 . Jahrhundert aufzuweisen hat. Aber ebensowenig dürfte dieser Absicht ein Vergleich mit dem System Hegels dienen, wozu der Gleichklang einiger Haupttermini verleiten könnte. Gegen die blendende Fassade der Manifestation des sich selbst begreifenden Geistes gehalten, erschiene Schleiermachers anthropologische Hermeneutik des Daseins als kümmerliche Aberration in dem geschichtlichen Entfaltungsproze $B$ der „Kategorien" der Weltvernunft. - Wir lehnen es ab, Schleiermachers Entwurf dem Endgültigkeitsanspruch Hegelscher Philosophie zu opfern. Hegels System ist in der Tat ein Ende, allerdings nur das Ende einer Periode, die mit der gott-losen Selbstgewißheit des Descartesschen cogito beginnt und sich über Kants Vernunft- und Postulatenreli- 
gion hinaus zur Seinslogik der ,metaphysischen Definitionen Gottes“ (Hegel. Enzykl. \& 85) entwickelt. Im „Zurücksehen " auf das Ganze der ,geistigen Gestaltungen der Philosophie" vermag es den Standpunkt Schleiermachers überhaupt nicht in den Blick zu bringen. Schleiermachers „Dialektik" ist ein neuer Ansatz der Denkbesinnung, ein neuer Traktat über die Methode, der aus dem Gefühl tiefster reformatorischer Gläubigkeit das Pseudochristentum Descartes' in die Schranken fordert. Es muß als fundamentaler Irrtum angesehen werden, wenn Hegel in den mit absolutem Seinsverlust verbundenen "Einklammerungen " und skeptischen Exerzitien der chose qui pense die Erscheinung des ",protestantischen Prinzips" christlicher Innerlichkeit erblickt (Hegel WW. Stuttg. I928 XIX, S. 328). Die Flucht in den ,reinen " Geist und in die bodenlose Selbstgewißheit eines daseinsmüden Bewußtseins hat nichts zu tun mit dem kämpferischen Daseinstrotz protestantischer Prägung. Schleiermachers Kunst der Gesprächführung ist in ihrem Grundplan unmittelbare Fortsetzung eckehart-cusanischer Laienfrömmigkeit und Laienweisheit, die dem Leitspruch: sapientia clamat in plateis seinen umfassendsten Ausdruck geben will. Es gilt, die ganze ungeteilte Wirklichkeitsfülle, wie sie in der Alltäglichkeit der Rede aufbricht, zu bewahren und trotz aller skeptischen Innehaltung keinen Inhalt preiszugeberr; es gilt, Wehr und Waffen zu schmieden für den immerwährenden Streit der Rede, durch den das „Dasein des Denkens" erst seinen Lebensinhalt erhält, und zu protestieren gegen jeden Sprung in eine kampflose und sich vornehm als höhere Potenz des Denkens aufblähende Geistigkeit. Es geht um das Wissen, daß innerhalb des Spannungsraumes zwischen dem Chaos der Empfindungen und dem uns einwohnenden Sein des transzendenten Grundes jeder Gedanke verknüpft ist „mit der Totalität des Wahrnehmbaren und jede Wahrnehmung mit der Totalität des Denkbaren", so daß wir jederzeit von der Mitte aus, d. h. durch den Ausgang von einer problematischen Tatsächlichkeit zur Homologie der Rede und somit zur Übereinstimmung mit dem Seienden gelangen können und an keiner Stelle des Wissensfortschrittes gegen die unverbrüchliche Harmonie von Natur und Geist verstoßen.

Schleiermacher, Dialektik. 
Bei Descartes wird Gott, wie Leibniz gezeigt, nur ,ad speciem quandam sive pompam" herbeigezogen (Gerh. IV S. 358). Der mittels eines logischen Zirkels schlecht versteckte Atheismus zeigt das Zerstörungswerk des radikalen Zweifels, der den festen Grund des credere anzutasten vermochte, gerade weil, was Hegel wiederum nicht begreift, bei Descartes das Christentum „als Denken zum Bewußtsein“ kam. In Schleiermachers neuem Entwurf der Methode hat uns echt protestantische Frömmigkeit den allem skeptischen Zugriff entzogenen Glaubensgrund zurückgegeben. Gott ist jenseits aller Begrifflichkeit, ja jenseits aller Denkgrenzen. Er steht zu aller fortschreitenden Weltweisheit in unwandelbarem konstanten Verhältnis; er ist, wie beim Cusaner, als das quantitativ Ewig-Unverhältnismäßige (finiti et infiniti nulla proportio) absolutes transfinites $\mathrm{MaB}$ alles Geschöpflichen und terminus a quo alles Weltwissens. In dem ,konstanten Quotienten“", der das geringste Denken zum Immerhöher-Steigen in die Wahrheit aufruft, offenbart sich das einwohnende Sein Gottes, der doch ewig dem Denken wie dem Wollen ein deus absconditus bleibt und nur in der Einheit von Wahrheit und Gewissen seine mittelbare Repräsentation findet. Man hat Schleiermachers Ontologie des Begriffs und Urteils als Mangel nachgesagt, da $B$ sie, die sich in dem Korrelativitätsanspruch von Denken und Sein auf den Weg gemacht habe, das Absolute zu suchen, am Schluß doch resignieren müsse, da sie die Gottheit jenseits aller $\mathrm{Be}-$ griffs- und Urteilsgrenzen findet. Ich halte es vielmehr für einen Vorzug und einen Triumph dieses Seinsentwurfes, nicht nur selbst der Gefahr einer Verbegrifflichung des Höchsten entgangen zu sein, sondern auch mit einer unerhörten Schärfe des Begriffs alle Irrwege der Verweltlichung Gottes aufgedeckt zu haben. Die Auseinandersetzung über die dialektische Korrelativität von Welt und Gott reißt in nüchterner Sachlichkeit das Nichts des Gottesgrundes auf, um im Gefühl des frommen Selbstbewußtseins den Ort aufzuweisen, wo christliche Dogmatik ihre Grundlegung erfahren soll.

Nur unzulänglich kann des streng bemessenen Raums wegen angedeutet werden, was das Geheimnis der „Dialektik" an Eigentlichem und Einzigartigem birgt. Einer von 
tiefster Ehrfurcht getragenen hermeneutischen Forschung sei das Werk zu fruchtbarer Erschließung und zu lebendiger Förderung protestantischer Weltbesinnung in die Hand gegeben.

\section{Die Grundsätze derneuen Bearbeitung}

Bevor ich die aus dem Geist der „Dialektik“ sich ergebenden Editionsprinzipien entwickle, gehe ich kurz auf die Geschichte der Textgestaltung ein. Die erste Ausgabe ist von L. Jonas 1839 (Schleiermachers Werke III. 4. 2) besorgt worden. Die zweite Redaktion unternahm J. Halpern I903. Neue handschriftliche Funde wurden 1878 von Bruno Weiß mitgeteilt (Untersuchungen über Fr. Schleiermachers Dialektik. I. Teil. Zeitschrift f. Phil. u. phil. Kritik. Bd. 73. Beilage G. u. H. der Dialektik. Als Anhang [S. I-43 des Bandes]). Die Ausgabe von Jonas zeichnet sich im großen und ganzen durch Sorgfalt und Treue in der Wiedergabe der Texte aus. Ihre redaktionellen Mängel, durch welche das Werk das Gegenteil dessen geworden ist, was Schleiermacher unter dem Ideal einer "absoluten Architektonik“ vorschwebte, sind jedem Leser der Schrift zur Genüge bekannt. Halperns Unternehmen beruht überhaupt nicht auf ernsthafter Quellenforschung, dringt somit nicht einmal zu einer Revision des von Jonas edierten Textes vor, sondern stellt ein eigenmächtiges Exzerpt aus den verschiedensten Entwicklungsstadien dar, das er für ,eine vollständige, geschlossene Gestalt der Dialektik in ihrer reifsten Ausbildung" ausgibt (Einl. zur Dial. 1903. S. XXXIII). Was er unter "Reife der Ausbildung" versteht, hat er an anderer Stelle entwickelt (Archiv f. Gesch. d. Phil. Bd. XIV. S. 2 Io ff.). Danach stellt das Grundheft von 18I4, das Jonas in den Mittelpunkt rückt und auf welches sich Schleiermacher vierzehn Jahre hindurch bezog, die schwächste Form dar, da in ihr angeblich dualistische und kritizistische Tendenzen hervortreten, die im Widerspruch zum maßgebenden Identitätsprinzip stehen sollen. Erst der Entwurf von 1818 gehe auf den ursprünglichen „Monismus“ von I8I I (A) zurück, während der neue Ansatz von I83I (E) der Vollendung am nächsten stehe und daher zum Kern einer neuen Materialgruppierung und Materialauslese gemacht werden müsse. 
Grundsätzlich ist hiergegen zu sagen, da $B$ schon allein die Absicht einer Stadienvermengung mit moderner, ganzheitlicher Editionsauffassung unvereinbar ist. Dazu kommt, da $B$ die Antithese: Monismus-Dualismus viel zu primitiv ist, um dem oszillatorischen Gedankengewebe der Dialektik gerecht zu werden. Die schlimmsten Widersprüche sind durch Wehrung (Dialektik Schleiermachers. Tüb. 1922. S. I6of.) aufgedeckt worden. Niemals wieder ist Schleiermacher auf die Fassung von I8I I zurückgegangen. Die Niederschrift von I83I stellt, soweit sich dies aus den vorhandenen Trümmern überhaupt feststellen läßt, gegenüber der klaren Gliederung von 1822 in redaktioneller Beziehung einen entschiedenen Rückgang dar, weicht aber inhaltlich in keinem wesentlichen Punkt von dem 1822 kernhaft entwickelten Programm ab. Am tollsten hat es Halpern mit dem formalen Teil getrieben; hier sind 130 Paragraphen des Textes von 1814 , also die gesamte Begriffs- und Urteilstheorie, der Schere zum Opfer gefallen. - Die Nachträge von Weiß sind für die Geschichte der Einleitung letzter Hand wichtig und finden im Anhang dieser Ausgabe ihre Berücksichtigung.

Jedenfalls zeigt Halperns mißglückter Versuch, daß sich mit noch so geistreichen Verschiebungen und Streichungen des von Jonas übernommenen Materials keine neue Ausgabe rechtfertigen läßt. Auch Wehrungs Versuch, einige Entwürfe Schleiermachers gesondert zu bringen und die Fassungen von $1814-1828$ synoptisch in Kolumnen nebeneinander $z u$ ordnen (Dial. Schleiermachers S. 6), ist aus den angegebenen Gründen nicht annehmbar und würde außerdem an buchtechnischen Schwierigkeiten scheitern.

Nun dürfte zunächst über das Oszillationszentrum des Werkes nach meinen Darlegungen kein Zweifel mehr bestehen. Die Stadienentwicklung der Entwürfe vollzieht sich unter zunehmender Dominanz des Postulates, da $B$ zwischen Denken und Reden der innigste Zusammenhang besteht, dem auch das sog. ,reine“ Denken unterliegt; und daß Dialektik, ihrem eigentlichen Wortsinn entsprechend, als K un st der Gesprächführung zu begründen ist. Wir als die Redenden sind das zunächst und vordringlich Wirkliche; in der communicatio des Gesprächs erschließt sich uns unser Menschsein, in dem scheiternden Streit des Dialogs die 
Wirklichkeit der Transzendenz. Das ist der ruhende Pol in der Flucht der verschiedenen Ansätze und Entwürfe, der bereits im Brouillon zur Ethik-Vorlesung von 1805 die Richtung der Dialektik als „,fortgesetztes Vergleichen einzelner Akte des Erkennens durch die Rede, bis ein identisches Wissen herauskommt", bestimmt (Sittenlehre. Ausg. O. Braun. S. $161-165)$. Bis zur endgültigen Klärung sind indessen noch viele Schwierigkeiten zu überwinden. I8II wird zwar angedeutet, daß kein Denkakt ohne organische Produktion sei (Dial. Jon. S. 315), aber ,die in allen eine und dieselbige Vernunft" läßt den Ausgang vom Geschehen der Rede noch abenteuerlich und aussichtslos erscheinen. Hier wie auch .1814 bleibt es bei der farblosen Definition der Dialektik als einer „Kunst des Gedankenwechsels“ (Jon. S. I7). Die Vorlesung I8I8 geht gelegentlich auf das Thema ein (Jon. ebda. u. S. 364), doch fehlt es auch jetzt noch an zielbewußter methodischer Auswertung. Erst das Jahr 1822 bringt die entscheidende Wendung. Unter der eindringlichen Beschäftigung mit der Hermeneutik ergab sich mit zunehmender Klarheit, daß das Gespräch das eigentliche Geschehen der Sprache sei und die Dialektik nur in wagemutigem Zupacken des Dialogos-Problems gedeihen könne. Daher denn auch der neue Vorlesungsentwurf, der den Rahmen des früheren sprengt und nur noch in lockeren Paragraphenverweisungen die Fäden zum alten Gespinst von 18I4 hinüberwirft. Soviel steht fest: der Keimgedanke des Dialogos ist mit 1822 souverän geworden; auf ihn hin muß das alte Gewebe mühsam aufgedröselt und umgesponnen werden. Die zunächst am Rande des Entwurfs von 1822 stehenden und später zum Heft von 1814 hinüberspringenden Notizen von I828 setzen dieses Geschäft fort. $\mathrm{Ob}$ allerdings mit der gleichen Energie und Konsequenz, läßt sich nicht feststellen, da Nachschriften aus diesem Jahre nicht vorliegen und die Bruchstücke bei Jonas kein entscheidendes Urteil zulassen. Doch ist auch hier die These, daß Denken die Geistestätigkeit sei, ,,welche sich durch die Rede vollendet" (Jon. S. 448), die richtunggebende Dominante gewesen. Das gleiche gilt von dem Entwurf des Jahres 1831, in dem der Satz: „Das Sprechen ist das Dasein des Denkens" (Jon. S. 492) das Leitmotiv aufklingen läßt, das 
dann in der Einleitung letzter Hand mit einem mächtigen Fortissimo die Darstellung intoniert.

Für die Edition ergeben sich hieraus unabweisliche Bindungen. Der Herausgeber ist nicht Vollzieher eines philologischen Geschäfts, durch das ein Konvolut von verstaubten Zetteln zur allgemeinen Kenntnis gebracht wird, sondern Vermittler und Neugestalter eines philosophisch bedeutsamen Sprachgeschehens. Was Schleiermacher von jeder philosophischen Rede verlangt: da $B$ sie sich im Gegensatz zum undialektischen Satz als Selbstgespräch in einem fortgesetzten Prozeß von "Hemmungen des reinen Denkens“ entwickelt, kann er mit Fug und Recht von der Wiedergabe seiner „Dialektik“ fordern. Nicht die Paragraphen von I8I4 dürfen den Kern der Dialektik bilden. Sie stellen noch chaotisch geballte Energien dar, für Schleiermachers Privatgebrauch bestimmt, um aus ihnen dem Geschehen des Selbstgespräches immer neue Nahrung zu geben. Wenn irgendeine der von Schleiermacher behandelten Disziplinen nach der Rekonstruktion des mündlichen Vortrags verlangt, so ist es die auf dem Boden dieses Postulates überhaupt erst lebensfähige Dialektik als Gespräch des Gesprächs. Erst, wenn wir in das gefügehafte Ganze solcher Vorlesung Einblick nehmen, erleben wir, wie einzigartig der Redner die regressive Methode der „Hemmungen“, des immer wieder auf den Anfangspunkt Zurückgehens, meistert und eine wie große Sünde am Geiste Schleiermachers es ist, dieses Gefüge in eine Summe von Teilzitaten zu zerstückeln.

Die Entscheidung für das Vorlesungsmaterial von 1822 ist damit aus systemimmanenten Gründen gerechtfertigt. Weitere Momente treten hinzu, um seine Bedeutung zu erhöhen. Die Vorlesung von 1822 setzt unmittelbar nach Beendigung des dogmatischen. Hauptwerkes ein und wird somit zu einer ,wichtigen Urkunde“, die ,einen unverfälschten Einblick in die rein begrifflichen Grundlagen des theologischen Systems" gewährt (Wehrung, Dial. Schleiermachers. S. I62). Sie vermittelt ein tieferes Verständnis für das Wesen des religiösen Gefühls, worauf ich an anderer Stelle hingewiesen habe (Das Gefüge des religiösen Bewußtseins bei Fr. Schl. Blätter $f$. deutsche Philos. Bd. 8 . S. 284-30I). Vor allem aber ist zu beachten, wie stark ge- 
rade in diesem Jahr das Erlebnis des Sprachgeschehens in Schleiermachers geistigem Schaffen gewesen ist. Der Vorsatz, die über ein Jahrzehnt liegengebliebene Arbeit am platonischen Dialogos wieder aufzunehmen (vgl. Leben i. Br. IV. An de Wette v. 17. Aug. 1822), erhält seinen Anstoß durch die gleiche Idee, nach der sich auch die in diesem Sommer gleichzeitig gehaltenen Vorlesungen über Dialektik und Hermeneutik zu einer schöpferischen Einheit gestalten sollten. Es erübrigt sich nach Diltheys aufschlußreichem Aufsatz (Die Entstehung der Hermeneutik. Ges. Schr. V. S. 317 ff.), auf die Verdienste Schleiermachers um die Begründung einer philosophischen Auslegekunst einzugehen. Bedeutsam für uns ist, daß sich im Verlauf dieser beiden Vorlesungen bei Schleiermacher das Gefühl des wechselseitigen Angewiesenseins beider Disziplinen immer mehr verstärkt, womit die Aufgabe der Dialektik erst ins rechte Licht gesetzt wird. Hermeneutik ist der Gegenpol zur Dialektik. Beide Disziplinen als Fundamentallehren von der Wirklichkeit des menschlichen Seins - „seit ein Gespräch wir sind und hören können voneinander" - zu begründen, ist Schleiermachers Lebensaufgabe. Das Heft von Saunier, das zugleich mit der Dialektik auch eine Ausarbeitung der Hermeneutik-Vorlesung desselben Jahres enthält, bietet eine Fülle von Beispielen für diese umgreifende Polarität. „Rede und Verstehen stehen immer im Verhältnis zueinander, und beiden liegt der gedachte Zusammenhang zugrunde; je besser gedacht, desto besser verstanden. Dialektik und Hermeneutik gehen also hier miteinander" (Heft Saunier; vgl. auch Schl. Hermeneutik. WW. I. 7. S. II). Diese Polarität ist eine so innige, daß die hermeneutische Situation nicht erst nach Abschluß der Rede einsetzt, sondern als immanente Gegeninstanz jeden Moment des dialektischen Prozesses beim Bewegen des Wortes in Beziehung auf die Sinntotalität des Sprachkreises durchwirkt (vgl. Schl. Herm. S. 10).

So fordert das Studium der Dialektik auch zugleich das der Hermeneutik. $\mathrm{Da} B$ dieser Forderung mit dem von Lücke edierten Material (WW. I. 7) nach Diltheys Urteil noch immer „nur in einer sehr unwirksamen Form" entsprochen 
werden kann, gehört in das Kapitel der Gleichgültigkeit gegenüber dem geistigen Vermächtnis des Mannes.

Mit alledem übernimmt der Herausgeber der Dialektik eine große Verantwortung. Dialektik und Hermeneutik sind polar gekoppelt. Dialektik als Gespräch des Gesprächs fordert zu ihrer Verlebendigung in gesteigertem $\mathrm{Maße}$ alle Kautelen heraus, die Hermeneutik und Kritik als bindend festgestellt haben Mag auch Schleiermacher die Kunst der hermeneutisch-dialektischen Rede meisterhaft geübt haben, so ist doch mit der Heranziehung der Nachschriften die Gefahr einer Sinnentstellung gegeben, die niemals völlig beseitigt werden kann, weil nicht überall der von Schleiermacher in das lebendige Wort gelegte Ausdruck dem Hörer „,in der Totalität seines Sprachwertes bekannt" war (Herm. S. 5I). Lücke hat in seiner Einleitung zur Hermeneutik bemerkt, daß es nicht leicht gewesen sein müsse, ,bei Schleiermacher ein gutes, vollständiges Heft zu schreiben", und daß sein Vortrag überwiegend so eingerichtet war, ,da $B$ er mehr zu einer freien Auffassung und Nachbildung als zu einem wörtlichen Nachschreiben veranlaßte" (Herm. S. IX). Das individuelle Moment des Nachschreibers auf ein Minimum zu reduzieren, ohne dabei im auswählenden Zugriff der eigenen Meditation zuviel Spielraum zu geben, d. h. die komparative und divinatorische Methode der Hermeneutik mit den Kautelen der Kritik, wie sie Schleiermacher gleichfalls entwickelt hat, zu vereinigen, ist oberstes Gebot, dessen Erfüllung aber nicht zuletzt von der Beschaffenheit des Quellenmaterials abhängt. Durch eine glückliche Fügung sind wir nun in den Besitz von vier Heften desselben begnadeten Jahres gekommen, von denen jedenfalls drei den Vortrag in seiner ganzen Entfaltungsbreite wiedergeben. Bei dem vierten herrschen zwar in der Einleitung und im formalen Teil stärkere Zusammendrängungen vor, doch kann es im transzendentalen Teil gleichfalls als authentische Unterlage benutzt werden. Dazu gesellen sich die Vorlesungsnotizen Schleiermachers aus demselben Jahre (C) und als weitere Kontrollinstanz einige von Jonas an verschiedenen Stellen seiner Ausgabe gebrachte Zitate nach dem Heft von Klamroth (I8 Abschnitte, die im kritischen Apparat dieser Ausgabe an den entsprechenden Stellen nam- 
haft gemacht werden). Auf Grund dieses recht reichhaltigen Materials habe ich das Wagnis einer Rekonstruktion unternommen, wobei ich mich, wie die Textvergleichungen zeigen mögen, der gewissenhaftesten Sachlichkeit befleißigt habe. Es braucht kaum erwähnt zu werden, daß alles vermieden ist, was als unkritische Verschönerung, eigenmächtige Modernisierung des Stils oder gar gewaltsam interpretierende Korrektur des Satzgefüges anzusehen ist. Natürlich konnte weder auf eine sich in gehörigen Grenzen haltende Konjekturalkritik noch auf behutsam vorgenommene Stilglättung verzichtet werden. Es verhält sich mit der Konjekturalkritik ähnlich wie mit der Dialektik. Auch für sie gibt es nach Schleiermacher „keine positiven Regeln, sondern nur Kautelen" (Herm. I. 7. S. 338). Das Wichtigste ist, „da $\beta$ die Konjektur der hermeneutischen Operation genügen“ muß (Herm. S. 340), d. h., daß ,das Verhältnis zwischen der Meditation und Komposition" des Redners deutlich hervortritt (vgl. Herm. S. I 53 u. 205). Maßstab für die Güte der Rekonstruktion sind solche schwierigen Stellen, wo Schleiermacher selbst noch nicht zur absoluten Klarheit zwischen Meditation und Komposition durchgedrungen war und dem Nachschreiber ein fast übermenschliches $\mathrm{Ma} B$ von Divinatorik zumutete, um, die Gedankenbewegung antizipierend, die Komposition verständnisvoll wiederzugeben. In solchen Fällen habe ich stets durch Abdruck aller in Frage kommenden Parallelstellen im Anhang'Rechenschaft abgelegt. Stilistische Änderungen nahm ich nur dort vor, wo offensichtliche Unbeholfenheit des Nachschreibers zutage trat. In jahrelangem Umgang mit dem Schrifttum Schleiermachers glaube ich die Fähigkeit erlangt zu haben, die Eigentümlichkeit seiner Ausdrucksform von der Unfertigkeit des studentischen Protokolls unterscheiden zu können. Varianten bedeutsamer Stellen sind in großer Auswahl gebracht worden und im Anhang nachzulesen. Wiederholungen im Vortrag Schleiermachers habe ich niemals gekürzt oder zusammengedrängt, weil sie ein wesentliches Moment im Oszillationsproze $B$ der Rede darstellen und stets Anlässe zu neuen spekulativen Impulsen sind. Die Freiheit, die sich Jonas bei der Behandlung der Kolleghefte nehmen konnte, „damit, was entzückte, wenn man es hörte, wenig- 
stens erträglich sei, wenn man es liest" (Christl. Sitte. 2. A. S. XIII), durfte von mir nicht in Anspruch genommen werden. Ich tröste mich mit der Hoffnung, der Leser möge das Gedruckte so lesen, als ob er es höre, damit es ihn von neuem entzücke, d. h. er möge sich der Geistesart Schleiermachers anzupassen suchen, der es mit der "Gewohnheit der Alten" hielt, ,,eine Rede zu hören“" und mehr fürs $\mathrm{Ohr}$ als fürs Auge zu schreiben (Hermeneutik. Heft Saunier). - So will also die vorliegende Ausgabe als dokumentarischer Bericht der dialektischen Leistung Schleiermachers gelten, wie sie zur Zeit seines fruchtbarsten Schaffens als lebendiges Geschehen hervorgetreten ist.

\section{Das handschriftliche Material}

a) Schleiermachers Niederschriften

Es folgt hier zunächst eine Übersicht über das gesamte handschriftliche Material zur Dialektik, dassich im Schleiermacher-Archiv der Preußischen Akademie der Wissenschaften befindet und zum großen Teil von Jonas für seine Ausgabe benutzt worden ist. Zu seiner Kennzeichnung bediene ich mich der von Jonas eingeführten Signierung.

A. Zettelnotizen für die Vorl. 18II. Sie sind chronologisch einwandfrei bestimmt (vgl. Br. Weiß, Untersuchungen über Fr. Schl.s Dial. Ztschr. f. Phil. u. phil. Kritik. Bd. 73. S. 21).

Grundheft I 8 I 4. Erste zusammenhängende, nach Paragraphen geordnete Niederschrift. Die Umschlagseite trägt die Aufschrift: Dialektik I8I4. Darunter: angefangen d. 24. Oct. 1814, geendigt d. 18 . März $1815 .-1818$ angefangen d. 19. Oct., geendet d. -1822 angefangen $d$. 15. April, in 5 Stunden wöchentlich. - Die fortlaufend numerierten Leitsätze sind außerdem mit römischen Ziffern versehen, durch welche die Stunden angegeben werden. $\mathrm{Zu}$ den Leitsätzen gesellen sich - anfangs vereinzelt (bei den $\S \S$ I6, I9, 21), von \& 86 ab fortlaufend - erklärende Korollarien, die von Schl. durch kleinere Schrift als solche namhaft gemacht sind. Aus der gleichmäßigen Ausfüllung des Zwischenraumes $z$ wischen den einzelnen Leitsätzen läßt 
sich schließen, daß das Ganze in einem Zuge hintereinander geschrieben ist. In vereinzelten Fällen sind wohl mehrere Leitsätze zunächst ohne Korollarien niedergeschrieben worden. Dafür spricht die Tatsache, daß der Zwischenraum an einzelnen Stellen nicht ausgereicht hat, so daß die erklärenden Zusätze hier und da am Rande fortgesetzt wurden. Es kann sich aber auch um Nachträge aus späteren Jahren handeln. In der Theorie der Urteilsbildung werden die Zusätze spärlicher. Von § 3I I ab fallen sie ganz fort. Zwischen den einzelnen Leitsätzen befinden sich größere Lücken, die nun nicht mehr ausgefüllt worden sind. Die Aufzeichnung der einzelnen Nummern hat nach der jeweiligen Vorlesung stattgefunden (vgl. Briefwechsel mit J. Chr. Gaß. Bln. 1852. S. I21).

B. Zettelnotizen für die Vorl. 1818 (vom 19. Okt. 1818 bis zum 24. März 1819), aus fünfzehn Blättchen bestehend. Ihre Datierung erweist sich aus der Übereinstimmung mit der Kollegnachschrift dieses Jahres. Sie bringen zu dem Grundheft von I8I4 nichts Neues hinzu und zeigen nur, daß sich Schl. in diesem Jahr fast ausschlieBlich auf dieses Heft bezieht, es nur hier und dort durch Randbemerkungen ergänzt. Einige geringfügige von Jon. übersehene Sätze sind von Weiß hinzugefügt worden (Weiß, ebda. S. I9).

C. (In der vorliegenden Ausgabe als laufender Begleittext zur Vorl. unter dem Strich gebracht.) Neue zusammenhängende Niederschrift für die Vorl. I822 (vom I5. April bis zum 16. Aug. 1822), die sich paragraphenweise auf das Grundheft von 1814 bezieht. Auch sie führt eine mit den Vorl. übereinstimmende römische Numerierung, bricht aber mit Vorl. LIX ( $=\S 234$ des Grundheftes) ab. Unter den Schluß sind die Worte gesetzt: Soweit war ich 1831 gekommen in 6r Stunden. Dadurch wird zum Ausdruck gebracht, daß auch noch 1831 , wo ein neuer Ansatz (E) erfolgt, der Text von $C$ und somit auch der des Grundheftes wenigstens zum Vergleich herangezogen worden sind. Es schließen sich dann in der ganzen Breite des Blattes die Notizen No. 62 bis 82 der Vorl. von 1831 an, die also zugleich als Ergänzung der 1822 nicht niedergeschriebenen Theorie der Begriffs- und Urteilsbildung gedacht sind. 
D. Fortlaufende Notizen zur Vorl. 1828, nach Stunden numeriert. Datierung außer Zweifel (vgl. Weiß S. 2I). Sie stehen am Rande von $C$ und brechen auch an der gleichen Stelle des formalen Teiles ab (mit der 59. Stunde). Ihre Fortsetzung finden sie mit der 68. Stunde am Rande des Grundheftes von 1814 (s. unten unter „Randbemerkungen“).

E. Zettelnotizen für die Vorl. I831 in der von Jonas und Wei $\beta$ vorgefundenen Verfassung. Der formale Teil wird auf den leeren Blättern von $C$ entwickelt (s. oben).

F. (In der vorliegenden Ausgabe auf S. I-44 wiedergegeben. Über den Zustand s. Anhang I dieser Ausgabe S. 467 ff.).

G. Ein von Weiß aufgefundenes und veröffentlichtes Heft (Weiß, Anhang, S. I-18) mit gelegentlichen Einfällen auf lose ineinandergelegten Bogen. Der Umschlag trägt die Aufschrift: Zur Dialektik I 8 I 4. Weiß liest diese Zahl irrtümlich als I 8I I und glaubt, da die ersten Notizen unzweideutig auf die Jahre 1814 und 1818 verweisen, wenigstens den Rest Nr. 97-I72 auf I8I I beziehen zu müssen. Äußerlich gesehen wäre diese Annahme insofern möglich, als mit Nr. 97 ein neuer Bogen beginnt, der später hineingelegt sein kann. Darüber hinaus äußert Wehrung die Vermutung: (Dial. Schl.s S. 8), daß das Mittelstück (4I-83) noch hinter die erste Fassung zurückzuverlegen sei. Diese Ansicht ist unannehmbar, weil der betreffende Bogen nicht mit Nr. 4I, sondern mit Nr. 40 beginnt.

H. Eine Reihe von Zetteln und Bogen, die Vorarbeiten zu $\mathrm{F}$ betreffend. Von Weiß durchgesehen und veröffentlicht. (In Anhang I der vorliegenden Ausgabe, S. 470 ff., ausgewertet.)

b) Die Randbemerkungen

Das Grundheft ist mit einer großen Zahl von Randbemerkungen versehen, deren Datierung große Schwierigkeiten gemacht hat (vgl. Weiß a. a. O. S. 22 ff. u. Wehrung, Dial. Schl.s S. $6 \mathrm{ff}$.). Jonas hat bei einigen das Entstehungsjahr 
durch Vergleich mit den Nachschriften feststellen können. Die von Jonas auf 1818 gehenden Datierungen konnten von mir nachgeprüft und bestätigt werden. Wichtig ist die Entscheidung über die Bemerkungen, die bei $\S 256$ (Jonas S. 202) beginnen und sich dann mit einigen Unterbrechungen von $\S 258$ bis $\& 330$ erstrecken. Jonas hat für ihre Datierung die Jahreszahl 1828 angegeben. Er unterließ es indessen, die ihnen von Schl. beigegebenen Stundenzahlen (68-74 und 76) hinzuzufügen, worauf Weiß aufmerksam gemacht hat. Auf Grund der vorhandenen Hefte lieB sich einwandfrei feststellen, da $B$ diese Stundenzahlen weder mit dem Verlauf der Vorl. von 1818 noch von I822 übereinstimmen. I831 kommt nach der Niederschrift E gleichfalls nicht in Frage. Es handelt sich also unzweifelhaft um eine Fortsetzung der Vorlesungsnotizen von 1828 (D), die am Rande von C. mit der Vorl. 59 abbrechen. Schl. bediente sich dann offenbar zunächst wieder des Grundheftes und trug dort von der 68. Stunde ab seine veränderten Gedanken ein.

c) Die Kollegnachschriften

An öffentlicher Stelle werden sechs Dialektik-Nachschriften aufbewahrt. Davon befinden sich vier im Schleiermacher-Archiv Berlin, eine in der Universitäts-Bibliothek Bonn, eine in der Universitäts-Bibliothek Göttingen. Für die vorliegende Ausgabe wurde nur das Material aus dem Jahre I 822 herangezogen. Doch sind vom Herausgeber auch die Kolleghefte von $1818 \mathrm{zu}$ einem Ganzen verarbeitet worden, um den Kern eines späteren Ergänzungsbandes zu bilden.

Für die Vorlesung I 8I 8/ I 9

I. Heft $\mathrm{Z}$ ander (Schl.-Archiv). Ein graumarmorierter Pappband mit Rückenschildchen. 378 beschriebene Seiten, die Seite mit 33-38 Zeilen, die Zeile mit $17-20$ Silben. Titel: Dialektik, vorgetragen von Fr. Schleiermacher im Winter 1818/19. Oben rechts die. Notiz von Jonas: v. Herrn Prediger Sander in Biesdorf erhalten d. 4. April 35 (vgl. Dial. Jon. S. XI). Stempel des Literaturarchivs. Sehr ausführliche, zusammenhängende Darstellung in ziemlich schwer 
lesbarer, abgekürzter Schrift. Keine Stundenmarkierungen. Auf den Rändern der Seiten eine große Zahl von Zusätzen.

2. Heft Friedrich Bluhme (Univ.-Bibl. Bonn. Signatur: S 839). Ein $31 \mathrm{I}+270$ Seiten umfassender Halblederband, der im ersten Teil die Dialektik von 1818/19, im zweiten Teil die Ästhetik Schl.s von I8I9 enthält. (Näheres in meiner Ausgabe der Ästhetik Schl.s. Berlin 193I. S. XXXI.)

\section{Für die Vorlesung I 822}

I. Heft Klamroth (Carl Heinrich Ludwig Kl. aus Parlin b. Stargard i. Pommern. Vater Prediger. An der Univ. Berlin inscr. v. 8. 4. I820-17. 8. I822). (Schl.-Arch.) Heft mit graublauem Umschlag ohne Rücken in Quart. 316 beschriebene Seiten; die Seite mit 32-37 Zeilen, die Zeile mit 17-25 Silben. Titel: Dialektik, gelesen von Professor Schleiermacher. Berlin, im Sommerhalbjahr I822. H. Klamroth. Am Ende der letzten Stunde: Geschlossen den I6. August 1822. Die einzelnen Stunden heben sich deutlich in Schrift und Tintenfärbung ab. Der Text ist lückenlos und gewissenhaft ausgearbeitet. Keine Vorl. fehlt. Einige $\mathrm{Zu}-$ sätze am Rande. Ausgiebige Verwendung von Sigeln und Wortkürzungen, die leicht entzifferbar sind. Das Heft stand Jonas zur Verfügung (vgl. Dial. Jon. S. X) und diente ihm als Unterlage für die Vorlesungszitate aus diesem Jahr. Die von Jonas zitierten Stellen sind im Anhang dieser Ausgabe namhaft gemacht. Die Textverkürzungen in den Zitaten stammen von Jonas, nich t vom Nachschreiber her. Klamroth wird in der Ausgabe der Pädagogik Schleiermachers von Platz als Superintendent tituliert.

2. Heft Kropatscheck (Schl.-Arch.) (Johann Gustav Wilhelm Kr. aus Nowawes b. Potsdam. Vater Prediger. An der Univ. Berlin inscr. v. 5. 4. 1820-7. 9. 1825). Graumarmorierter Pappband mit rotem, bedrucktem Rückenschildchen. Quartformat. 197 beschriebene. Seiten; die Seite mit 34-39 Zeilen, die Zeile mit 27-35 Silben. Auf dem Vorsatzblatt Name des Nachschreibers mit Angaben über den Einband. Dazu von späterer Hand Hinweis auf die Ausgabe von Jonas (1839) und auf die Rezension dieser Ausgabe 
durch Weiße in den Jahrbüchern für wissensch. $\mathrm{Kr}$. 1839 . Titel: Die Dialektik, vorgetragen von H. Professor Schleiermacher im Sommersemester 1822. J. Kropatscheck. Die einzelnen Stunden sind mit genauem Datum versehen. Der Text ist lückenlos und in sauberer Schrift ausgearbeitet. Einige spätere Zusätze am Rande. Verwendung von leicht lesbaren Sigeln und Abkürzungen.

3. Heft Saunier (Schl.-Arch.) (Johann Carl Heinrich S. aus Berlin. Vater Prediger. An der Univ. Berlin inscr. v. 17. 8. I821-8. I0. I822. Als candidatus alumnus verstorben. Schl. hielt die Grabrede [s. Schl.s Predigten. WW. II. 4. S. 869]). Graumarmorierter Pappband mit blauem Rückenschildchen. Quartformat. Enthält im ersten Teil die Dialektik von 1822 auf 184 Seiten, die Seite mit 28 Zeilen, die Zeile mit 13-17 Silben; im zweiten Teil die Hermeneutik. Titel des ersten Teils: Dialektik nach Schleiermacher, zum Theil in Auszügen im Sommer 1822. Darunter: durchgesehen und vervollständigt Frühjahr 1824. Am FuB des Blattes die Namen: Saunier und (mit anderer Hand) Schirmer (wohl der spätere Besitzer des Heftes). In der Schrift klar mit vielen Abkürzungen und Sigeln. Auf den Rändern einige spätere Nachträge und Kapitelüberschriften. Der allgemeine Teil, etwa bis Vorl. 22, wird in gedrängter Form, der transzendentale Teil ziemlich wortgetreu wiedergegeben. Sehr ausführlich ist der religionsphilosophische Abschnitt. Im formalen Teil zeigen sich wieder stärkere Zusammendrängungen.

4. Heft Szarbinowski (Univ.-Bibl. Göttingen. Standnummer: Cod. M. S. philos. $\mathbf{I}^{\mathrm{s}}$. Geschenk des Herrn Prof. D. E. Hirsch', Göttingen, der es am I4. 6. 1941 aus dem Buchhandel in Leipzig erworben hat). (Franz Ludwig Eduard S. aus Bromberg [Posen]. Vater Stadtverordneter. Bei der jurist. Fakultät d. Univ. Berlin inscr. v. 18. 8. 1821 bis 10. 4. 1823.) Braunmarmorierter Pappband mit rotem Rückenschild. Quartformat. 335 numerierte, beschriebene Seiten, die Seite mit 28-30 Zeilen, die Zeile mit 15-17 Silben. Titel: Grundzüge der Dialektik nach Prof. Schleiermacher. Berlin, d. 15. April I822. E. Szarbinowski. Am 
Schluß des Heftes das Datum: I6. August 1822. Auf dem Vorsatzblatt ein Vermerk von Prof. Hirsch (Juni 194I) über Qualität der Nachschrift und eine Zusammenstellung der auf I822 bezogenen Vorlesungszitate in der Ausgabe von Jonas. Sorgfältige, gut lesbare, gleichmäßige Schrift mit einigen Sigeln und Wortkürzungen, die auf den ersten Seiten am Rande erklärt sind. Keine Stundenmarkierungen. Nicht überall gleichmäßig in der Qualität; hier und da starke Sinnentstellungen. Verschiedene Lücken.

\section{Erklärungen zur äußeren Textgestaltung}

Schleiermachers für den Druck bestimmte Einleitung (F) ist der Vorlesung von 1822 vorangestellt worden (S. I-44). Die Leitsätze sind durch kursive Schrift gekennzeichnet.

Das Textbild der Vorlesung von 1822 will das Geschehen der Rede in lückenloser Entwicklung wiedergeben. Schleiermachers Notizen aus diesem Jahr (Beilage C) mußten deshalb unter dem Strich der Vorlesung parallel geschaltet werden.

Die Einteilung des St of fes ergibt sich aus der von Schleiermacher selbst getroffenen Anordnung (Einleitung [wofür hier: Allgemeiner Teil], transzendentaler Teil, formaler oder technischer Teil). Weitere Einschnitte in den Fluß des Vortrags zu machen, widerstrebte Schleiermacher. Zum Zweck einer schnellen Orientierung des Lesers sind vom $\mathrm{Hg}$. in den laufenden Text abschnittweise Kapite 1überschriften eingestreut worden, deren Formulierung in einigen Fällen Schleiermachers eigenen Marginalien oder den Nachschriften entnommen werden konnte.

Die Paragraphenverweisungen in den Notizen Schleiermachers beziehen sich auf das Grundheft von 1814, das in der Ausgabe von Jonas vorliegt. Um die entwicklungsgeschichtlich vergleichende Arbeit zu erleichtern, sind auch den einzelnen Kapiteln im Text, soweit es angängig war, Paragraphenhinweise beigegeben worden; doch keineswegs im Sinne völliger Übereinstimmung. Schon die Ein- 
leitung von 1822 weicht erheblich von der Fassung der früheren Jahre ab, so da $\beta$ eine Bezugnahme auf die Paragraphen von 1814 anfangs nicht möglich war. Auch in der weiteren Entwicklung des Vortrags ergeben sich mitunter erhebliche Differenzen, die Umgruppierungen und Ausschaltungen von Paragraphen zur Folge haben. Im übrigen sind diese Verweisungen für das Verständnis der ganz auf sich gestellten Vorlesung ohne Bedeutung.

Sperrungen in der Vorlesung wurden vom $\mathrm{Hg}$. auf eigene Verantwortung vorgenommen. Sie betrafen vor allem solche Stellen, an denen der Rhythmus der Gedankenbewegung sich zu einer undialektischen Aussage in Form von Regeln und Kernsätzen verdichtet.

Die $Z$ ahlen neben den einzelnen Vorlesungen bezeichnen laufende Nummer und Jahrestag der betreffenden Stunde nach dem Heft von Kropatscheck. 
